05,01

\title{
Локализованные моды спин-волнового резонанса ферромагнитных микрополосок в поле магнитного зонда
}

\author{
(C) Р.В. Горев, В.Л. Миронов
}

Институт фозики микроструктур РАН, Нижний Новгород, Россия

E-mail: GorevRV@ipmras.ru

\begin{abstract}
Приводятся результаты микромагнитного моделирования вынужденных колебаний намагниченности планарных микрополосок из $\mathrm{NiFe}$ с анизотропией легкая плоскость и из $\mathrm{Co} / \mathrm{Pt}$ с перпендикулярной анизотропией типа легкая ось в поле магнитного сферического зонда. Показано, что под воздействием поля зонда в полосках $\mathrm{NiFe}$ реализуется связанное состояние „еж“-,,антивихрь“, обусловленное латеральными компонентами поля зонда, а в слое Сo/Рt реализуется скирмионное состояние намагниченности. Эти эффекты приводят к искажению пространственных распределений колебаний намагниченности микрополосок и к появлению дополнительных резонансов в спектрах колебаний, связанных с модами, локализованными в поле зонда.
\end{abstract}

Работа выполнена при поддержке Российского научного фонда (проект № 16-12-10254).

DOI: 10.21883/FTT.2017.11.45053.10k

\section{1. Введение}

В последнее время большое внимание уделяется исследованию локальных мод ферромагнитного резонанса (ФМР), обусловленных воздействием неоднородного поля зонда магнитно-резонансного силового микроскопа (МРСM) на тонкопленочные ферромагнитные образцы [1-3]. С одной стороны, такое воздействие зонда приводит к нежелательным искажениям характера колебаний намагниченности исследуемых объектов, однако, с другой стороны, в условиях сильного взаимодействия зонда и образца возможна реализация новых методик исследования локальных СВЧ-свойств ферромагнитных структур [4-6]. В настоящей работе методами микромагнитного моделирования исследованы особенности воздействия магнитного сферического зонда на колебания намагниченности тестовых образцов в виде тонкопленочных ферромагнитных микрополосок с анизотропией типа легкая плоскость и с перпендикулярной анизотропией типа „легкая ось“.

\section{2. Методика расчетов}

Микромагнитное моделирование проводилось на основе численного решения уравнения Ландау-Лифшица-Гильберта для намагниченности образца с использованием программного пакета Object Oriented MicroMagnetic Framework (OOMMF) [7]. В качестве тестовых объектов исследовались прямоугольные микрополоски с размерами $2000 \times 1000 \times 10 \mathrm{~nm}$. Расчеты проводились для двух различных систем. Первая представляла собой микрополоску из пермаллоя
$\left(\mathrm{Ni}_{80} \mathrm{Fe}_{20}\right)$ со следующими параметрами: намагниченность насыщения $M_{\mathrm{NiFe}}=8 \cdot 10^{5} \mathrm{~A} / \mathrm{m}$, обменная константа $A_{\mathrm{NiFe}}=1.3 \cdot 10^{-11} \mathrm{~J} / \mathrm{m}$. Вторая система представляла собой многослойную структуру $\mathrm{Co} / \mathrm{Pt}$ с перпендикулярной анизотропией, имеющую следующие параметры: намагниченность насыщения $M_{\mathrm{CoPt}}=8 \cdot 10^{5} \mathrm{~A} / \mathrm{m}$, обменная константа $A_{\mathrm{CoPt}}=10^{-11} \mathrm{~J} / \mathrm{m}$, параметр анизотропии $K=6 \cdot 10^{5} \mathrm{~J} / \mathrm{m}^{3}[8,9]$. В расчетах параметр диссипации составлял 0.01. В качестве поля модельного МРСМ-зонда было выбрано поле однородно намагниченного по оси $z$ шара из Со (рис. 1). Диаметр зонда соответствовал типичным размерам зондов, используемых в МРСМ [10,11], и составлял $200 \mathrm{~nm}$. Исследовались колебания намагниченности образцов в переменном магнитном поле $h$ величиной $0.1 \mathrm{mT}$, направленном в плоскости образца вдоль оси $y$. В расчетах регистрировались временные зависимости установившихся колебаний всех компонент намагниченности [12]. Частота возбуждающего СВЧ-поля v изменялась с шагом $0.1 \mathrm{GHz}$. Для анализа спектров колебаний строились частотные зависимости

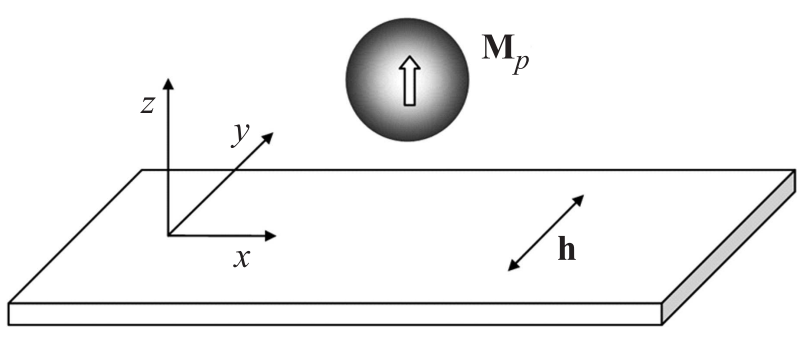

Рис. 1. Схематическое изображение магнитного зонда над ферромагнитной микрополоской. 

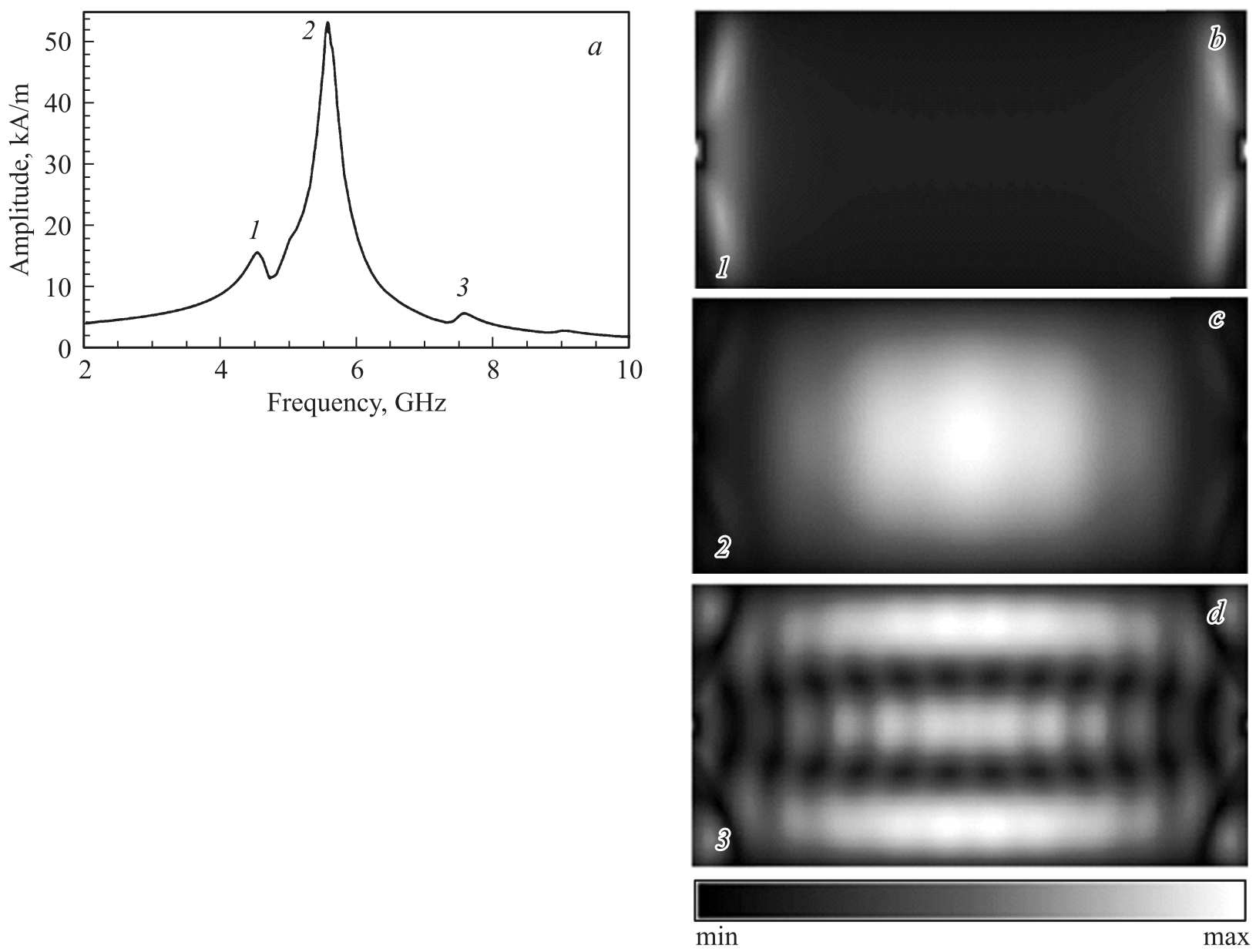

Pис. 2. (a) Спектр колебаний намагниченности однородно намагниченной микрополоски $\mathrm{NiFe} .(b)-(d)$ Нормированные пространственные распределения амплитуды колебаний намагниченности, соответствующие резонансным пикам $1-3$ в спектре рис. 2, $a$.

средней по системе амплитуды колебаний переменной составляющей намагниченности

$$
m=\sqrt{m_{x}^{2}+m_{y}^{2}+m_{z}^{2}} .
$$

Для анализа модового состава резонансов рассчитывались временные реализации пространственных распределений амплитуды колебаний переменной составляющей намагниченности при накачке на резонансных частотах $[13,14]$.

\section{3. Результаты и обсуждение}

Вначале нами исследовался ФМР в микрополоске $\mathrm{NiFe}$ в отсутствие зонда. Для создания однородного состояния к ней прикладывалось постоянное магнитное поле величиной $30 \mathrm{mT}$, направленное вдоль длинной стороны (вдоль оси $x$ ). Спектр колебаний намагниченности показан на рис. 2,a. Данный спектр имеет три характерных резонансных пика. Первый пик соответствует моде колебаний намагниченности, локализованной вблизи края микрополоски (рис. 2,b). Второй резонанс на частоте $5.5 \mathrm{GHz}$ представляет собой суперпозицию стоячих магнитостатических и обменных спиновых волн с различными волновыми векторами $k_{x}$ (рис. 2,c) [14]. Третий резонанс представляет собой суперпозицию стоячих спиновых волн с различными волновыми векторами $k_{x}$ и $k_{y}$ (рис. $2, d$ ).

При подводе зонда к образцу поле зонда вначале вызывает небольшие возмущения намагниченности микрополоски, а при достаточно малых расстояниях зондобразец $(<40 \mathrm{~nm})$ приводит к эффектам перемагничивания [15]. Как результат, под действием латеральных компонент поля зонда в микрополоске формируется неоднородное состояние „еж“-,антивихрь“" (распределение намагниченности на участке микрополоски в окрестности зонда приведено на рис. 3,a). Воздействие зонда приводит к сдвигу основного резонанса в низкочастотную область спектра (рис. 3,b). При этом к резонансу краевых мод добавляется низкочастотный резонанс антивихревого состояния (рис. 3,c), а также происходит деформация основной моды колебаний (рис. $3, d$ ) и спин-волнового резонанса (рис. $3, f$ ). Кроме того, в ФМР-спектре появляется дополнительный резо- 

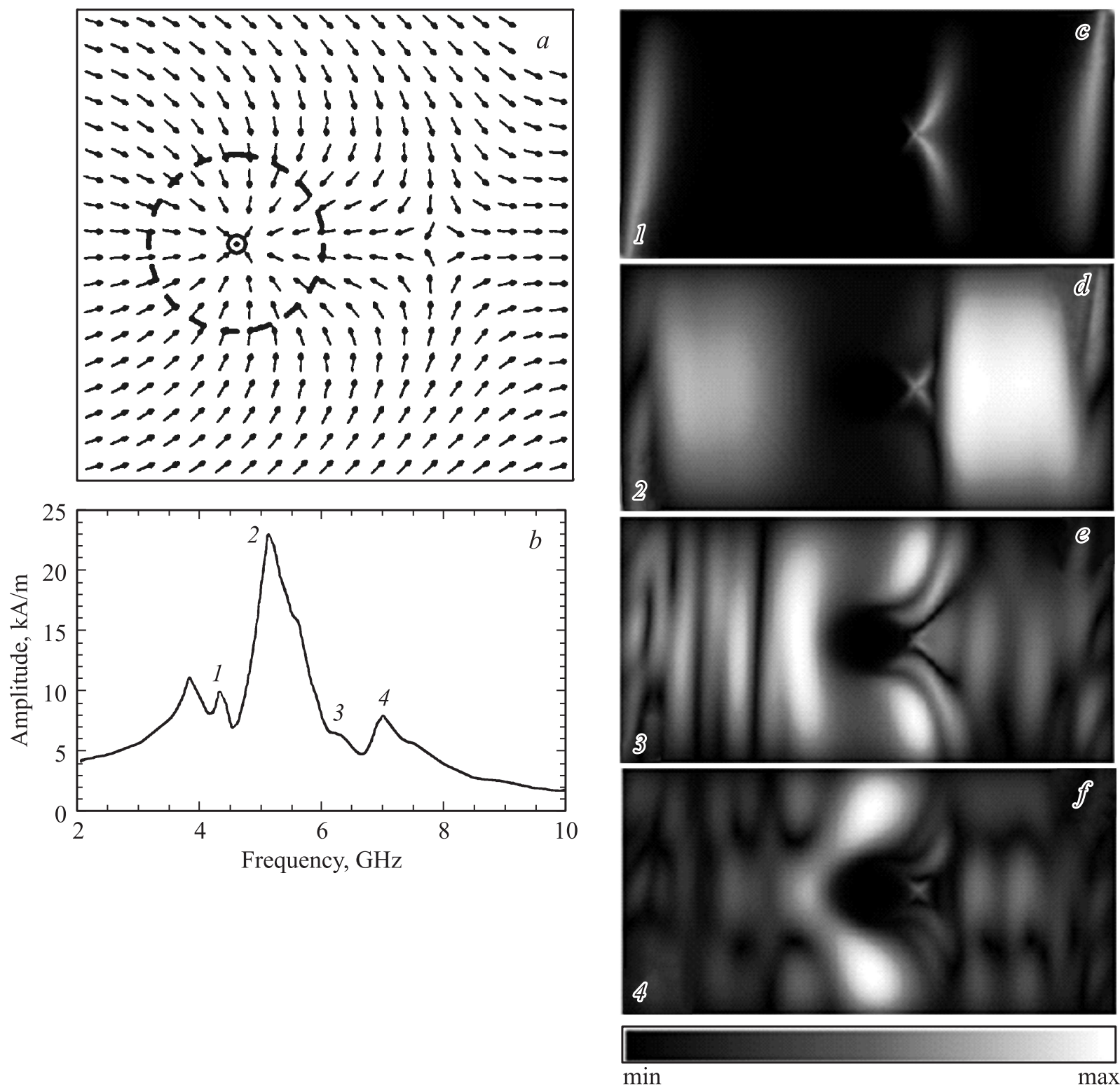

Pис. 3. (a) Распределение намагниченности на участке $(450 \times 450 \mathrm{~nm})$ микрополоски $\mathrm{NiFe}$ непосредственно в области под зондом MPCM. Положение зонда над образцом обозначено пунктирным контуром. (b) Спектр колебаний намагниченности полоски NiFe в поле зонда. $(c)-(f)$ Нормированные пространственные распределения амплитуды колебаний намагниченности, соответствующие резонансным пикам $1-4$ спектра на рис. $3, b$.

нансный пик 3, связанный с модой колебаний, показанной на рис. $3, e$.

Аналогичные эффекты наблюдаются в микрополоске $\mathrm{Co} / \mathrm{Pt}$ с перпендикулярной намагниченностью. В отсутствие зонда в однородно намагниченном состоянии спектр колебаний полоски содержит ряд резонансов различного порядка (рис. 4,a). Основной резонанс (пик 1) соответствует квазиоднородной прецессии намагниченности (рис. 4, $b$ ). Два другие являются спин-волновыми резонансами более высокого порядка (рис. 4,c,d). Поле зонда вначале вызывает небольшие возмущения намагниченности микрополоски, а при малом расстоянии между зондом и образцом приводит к эффектам пе- ремагничивания [16]. Для рассматриваемых параметров системы критическое расстояние составляло $40 \mathrm{~nm}$. При этом симметрия магнитного поля зонда способствует формированию в полоске $\mathrm{Co} / \mathrm{Pt}$ скирмионного состояния (рис. 5,a). Такое воздействие зонда приводит к сдвигу основного резонанса в высокочастотную область спектра (рис. 5,b) и к искажению пространственного распределения колебаний в основной моде (рис. 5,c). Кроме того, эффект перемагничивания приводит к появлению в спектре ФМР дополнительного интенсивного резонансного пика (пик 2, рис. $5, b$ ), связанного c модой колебаний намагниченности показанной на рис. $5, d$. 

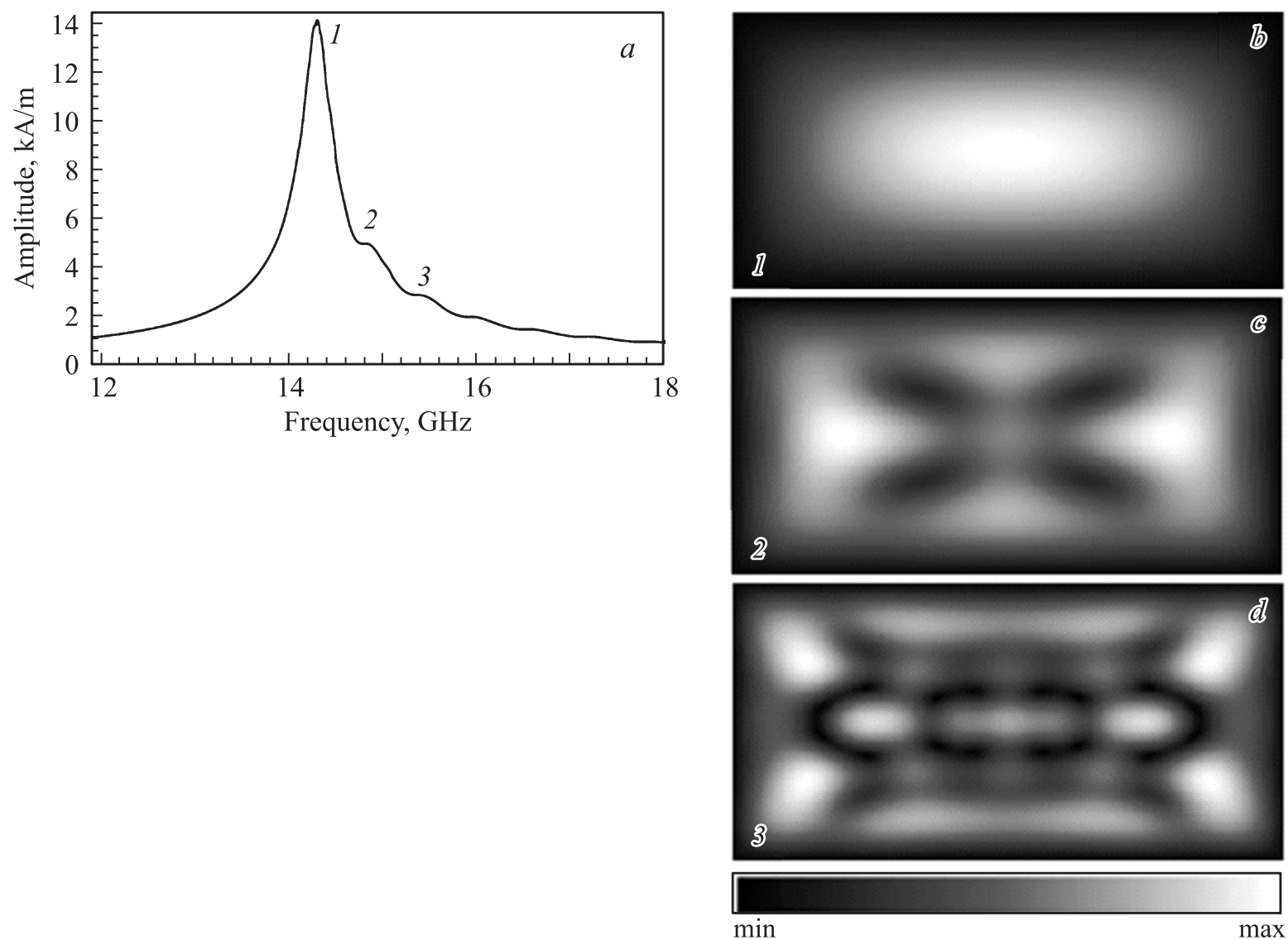

Pис. 4. (a) Спектр колебаний намагниченности однородно намагниченной микрополоски Co/Pt. $(b)-(d)$ Нормированные пространственные распределения амплитуды колебаний намагниченности, соответствующие резонансным пикам $1-3$ в спектре рис. $4, a$.
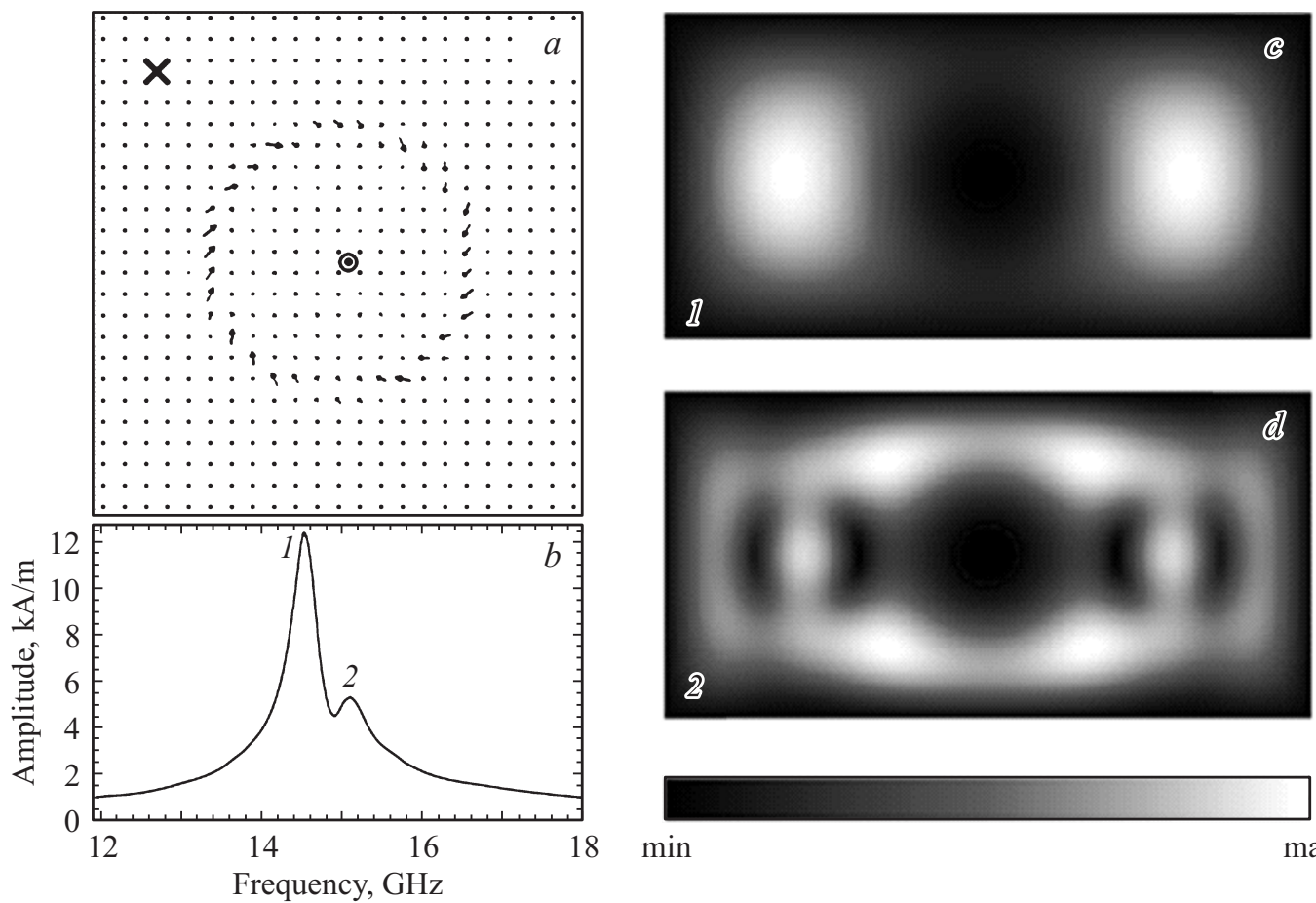

$\min$

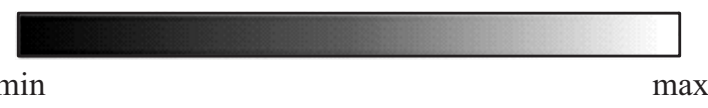

Рис. 5. (a) Распределение намагниченности на участке микрополоски Сo/Pt (размером $700 \times 700 \mathrm{~nm})$ непосредственно под зондом МРCM. Намагниченность в центральной области направлена вверх (в направлении оси $z$ ), а на периферии - в противоположном направлении. $(b)$ Спектр колебаний намагниченности полоски $\mathrm{Co} / \mathrm{Pt}$ в поле зонда. $(c)-(d)$ Нормированные распределения амплитуды колебаний намагниченности, соответствующие резонансным пикам 1 и 2 в спектре рис. $5, b$. 


\section{4. Заключение}

Таким образом, нами проведено микромагнитное моделирование эффектов влияния неоднородного поля магнитного зонда МРСМ на спектры и пространственную структуру мод колебаний намагниченности в микрополосках из $\mathrm{NiFe}$ с анизотропией типа легкая плоскость и микрополосках из многослойных структур $\mathrm{Co} / \mathrm{Pt} \mathrm{c}$ перпендикулярной анизотропией типа легкая ось. Показано, что при малых расстояниях зонд-образец наблюдается локальное перемагничивание образцов. При этом в полосках пермаллоя реализуется связанное состояние „еж“-,антивихрь“, обусловленное латеральными компонентами поля зонда, а в ФМР-спектре появляется дополнительный резонанс, связанный с локализованной модой колебаний намагниченности. В полоске $\mathrm{Co} / \mathrm{Pt}$ реализуется скирмионное состояние намагниченности, что сопровождается появлением дополнительного резонанса, связанного с колебаниями намагниченности в поле зонда. Данные эффекты необходимо учитывать при проведении МРСМ-исследований ферромагнитных пленок с различным типом анизотропии.

\section{Список литературы}

[1] A. Suter, D.V. Pelekhov, M.L. Roukes, P.C. Hammel. J. Magn. Res. 154, 210 (2002).

[2] I. Lee, Yu. Obukhov, G. Xiang, A. Hauser, F. Yang, P. Banerjee, D.V. Pelekhov, P.C. Hammel. Nature 466, 845 (2010).

[3] H.-J. Chia, F. Guo, L.M. Belova, R.D. McMichael. Phys. Rev. Lett. 108, 087206 (2012).

[4] I. Lee, Yu. Obukhov, A.J. Hauser, F.Y. Yang, D.V. Pelekhov, P.C. Hammel. J. Appl. Phys. 109, 07D313 (2011).

[5] C. Du, R. Adur, H. Wang, S.A. Manuilov, F. Yang, D.V. Pelekhov, P.C. Hammel. Phys. Rev. B 90, 214428 (2014).

[6] C. Du, I. Lee, R. Adur, Yu. Obukhov, C. Hamann, B. Buchner, J. McCord, D.V. Pelekhov, P.C. Hammel. Phys. Rev. B 92, 214413 (2015).

[7] M.J. Donahue, D.G. Porter. Interagency Report No. NISTIR 6376. National Institute of Standards and Technology, Gaithersburg. (http://math.nist.gov/oommf/).

[8] M. Yu, Y. Liu, D.J. Sellmyer. J. Appl. Phys. 87, 6959 (2000).

[9] S.H. Liou, S. Huang, E. Klimek, R.D. Kirby, Y.D. Yao. J. Appl. Phys. 85, 4334 (1999).

[10] F. Guo, L.M. Belova, R.D. McMichael. Phys. Rev. Lett. 110, 017601 (2013).

[11] H. Lavenant, V. Naletov, O. Klein, G. de Loubens, L. Casado, J.M. De Teresa. Nanofabrication, 1, 65 (2014).

[12] Р.В. Горев, В.Л. Миронов, Е.В. Скороходов. Поверхность. Рентгеновские, синхротронные и нейтронные исследования 3, 37 (2016).

[13] Р.В. Горев, Е.В. Скороходов, В.Л. Миронов. ФТТ 58, 2135 (2016).

[14] E.V. Skorohodov, R.V. Gorev, R.R. Yakubov, E.S. Demidov, Yu.V. Khivintsev, Yu.A. Filimonov, V.L. Mironov. J. Magn. Magn. Mater. 424, 118 (2017).

[15] В.Л. Миронов, О.Л. Ермолаева, А.А. Фраерман. Изв. РАН. Сер. физ. 72, 1558 (2008).

[16] V.L. Mironov, B.A. Gribkov, S.N. Vdovichev, S.A. Gusev, A.A. Fraerman, O.L. Ermolaeva, A.B. Shubin, A.M. Alexeev, P.A. Zhdan, C. Binns. J. Appl. Phys. 106, 053911 (2009). 